\title{
Qualidade de vida e funcionalidade na doença de Parkinson
}

\author{
Rafaela Silva Quintella*, Amanda Sachetti", Lia Mara Wibelinger"**, \\ Sheila Gemelli de Oliveira****
}

\section{Resumo}

A doença de Parkinson (DP) é uma doença neurodegenerativa crônica, cujo diagnóstico é estabelecido a partir dos sintomas clínicos, principalmente a rigidez muscular, tremor de repouso, bradicinesia e alteração postural, que afetam os aspectos da qualidade de vida e funcionalidade dos portadores dessa doença. Este trabalho teve como objetivo avaliar a qualidade de vida (QV) e a funcionalidade de pacientes com doença de Parkinson. Foram avaliados oito pacientes com DP da Clínica de Fisioterapia da Universidade de Passo Fundo. A pesquisa foi realizada no período de outubro a novembro de 2010. Para a coleta de dados, utilizou-se "Questionário PDQ 39, Medida de Independência Funcional (MIF) e uma ficha de avaliação neurofuncional. Os resultados obtidos por meio do PDQ 39 indicaram pior percepção da QV nas dimensões "mobilidade" e "atividade de vida diária". Na MIF, os resultados indicaram que $13 \%$ da amostra necessitam assistência de $50 \%$ na execução das tarefas, $38 \%$ da amostra apresentou independência completa e 49\% necessita de assistência de $25 \%$ na execução de tarefas. Diante da análise deste estudo, pode-se concluir que a doença de Parkinson traz ao paciente limitação funcional que interfere na qualidade de vida.

Palavras-chave: Doença de Parkinson. Qualidade de vida. Funcionalidade. Idosos.

\section{Introdução}

A doença de Parkinson (DP) é uma doença crônica e neurodegenerativa, com incidência na população acima de 65 anos, de $1 \%$ a $2 \%$ no mundo e prevalência de $3 \%$ no Brasil (LUNDY-EKMAN, 2000). O diagnóstico é feito de acordo com os sintomas clínicos, principalmente a rigidez muscular, tremor, bradicinesia e alteração postural (FINGER; PRADO; BOFF, 2003). Algumas implicações físicas vão se manifestando pelo decréscimo da força muscular e do condicionamento físico, além da ocorrência de um grande impacto no estado mental, social e econômico dos indivíduos portadores da

* Fisioterapeuta pela Universidade de Passo Fundo.

** Fisioterapeuta pela Universidade de Passo Fundo. Mestranda em Ciências Pneumológicas pela Universidade Federal do Rio Grande do Sul. Bolsista CAPES. Endereço para correspondência: Amanda Sachetti - Rua Harry Becker, 567 bairro Santa Maria, Passo Fundo/RS CEP 99070-190. E-mail: amandasachetti@gmail. com

*:** Docente curso Fisioterapia UPF / Doutora em Gerontologia Biomédica/PUCRS.

**** Docente curso Fisioterapia UPF / Mestre em Gerontologia Biomédica/PUCRS.

$\rightarrow$ http://dx.doi.org/10.5335/rbceh.2013.1823 
doença e de seus familiares. Diante da cronicidade e progressão dessa doença, a necessidade de apoio torna-se evidente, ressaltando o papel da família e de suas responsabilidades quanto aos cuidados. Nesse sentido, a qualidade de vida pode se tornar prioridade para os indivíduos diagnosticados com DP (TEIXEIRA; NERI, 2008).

Muitas doenças neurológicas devastadoras envolvem a morte lenta e progressiva de neurônios. Os indivíduos acometidos por essas doenças perdem mais de $80 \%$ dos neurônios que utilizam dopamina na substância negra (O'SULLIVAN; SCHMITZ, 2004; GOULART et al., 2005). A perda de influência dos neurônios dopaminérgicos leva à redução no movimento espontâneo, configurando um distúrbio motor importante e característico da DP (BEAR et al. 2008). Portanto, o estudo do sistema nervoso faz-se necessário para auxiliar na compreensão, cada vez mais consistente, das conexões multifacetadas que formam a base de cada ação que executamos, de cada sensação percebida, de processos de reconhecimento e de sentimentos como felicidade, tristeza e ciúmes (GUYTON, 2008; KLINKE; SILBERNAGL; BAUER, 2006). Na DP, essas ações poderão representar algumas das limitações ou incapacidades impostas pela própria doença (LUNDY, 2000; LENT, 2001).

Com a evolução da doença, complicações secundárias decorrentes dos sinais e sintomas físicos determinam o comprometimento mental, emocional, social e econômico, o que se revela extremamente incapacitante para o indivíduo, além de contribuir para a piora da qualidade de sua vida (LANA et al., 2007; DARABAS; COMIM; TUON, 2009).
A tendência ao estigma leva esses sujeitos a apresentem uma capacidade cognitiva desacreditada, fator que influencia diretamente nas interações sociais. Nesse momento, os tratamentos também devem priorizar aspectos importantes da qualidade de vida, principalmente no que se refere à saúde psicológica, reforçando a importância das relações interpessoais como fator determinante para atingir um envelhecimento o mais saudável e gratificante possível (FLORIANO; DALGALARRONDO, 2007).

A QV pode ser considerada como multidimensional, por refletir a avaliação subjetiva do sujeito com relação a todos os fatores já mencionados (POPE; NICHOLAS, 2009). A partir da constatação em estudos e pesquisas, disponibilizam-se meios para que a $\mathrm{QV}$ seja avaliada através de instrumentos genéricos, com o objetivo de verificar o impacto que a doença gera e, em termos de independência funcional, identificar as incapacidades que configuraram as restrições funcionais da pessoa, pelo impacto causado pela doença (LANA et al., 2007).

A fisioterapia, portanto, desempenha um importante papel na reabilitação, ou seja, na redução das limitações funcionais e das incapacidades, além da promoção da saúde, tornando possível a melhora no quadro motor, na independência funcional do paciente, no equilíbrio, além de fornecer orientações para favorecer a eliminação dos fatores de risco e promover a qualidade de vida (IKE; CARDOSO; BARALDI, 2008; PICKLES, 2000).

A hipótese gerada neste estudo é de que a $\mathrm{QV}$ e a funcionalidade de parkinsonianos são afetadas pela progressão 
da doença e o objetivo, portanto, foi avaliá-las em pacientes com doença de Parkinson.

\section{Materiais e métodos}

O estudo caracteriza-se por ser descritivo e exploratório. Sua população foi constituída de oito pacientes com diagnóstico clínico de doença de Parkinson, de ambos os gêneros, com idades variando entre 59 e 94 anos, atendidos pela Clínica de Fisioterapia da Universidade de Passo Fundo. A amostra foi selecionada a partir dos seguintes critérios: apresentar comprovação da doença mediante laudo médico; aceitação prévia dos pacientes com assinatura do Termo de Consentimento Livre e Esclarecido. Os estágios da doença não foram considerados, e os pacientes deveriam estar recebendo tratamento fisioterapêutico. Todos os sujeitos da amostra foram esclarecidos quanto aos objetivos da pesquisa, a qual foi aprovada pelo Comitê de Ética em Pesquisa da Universidade de Passo Fundo sob o registro no CEP, n ${ }^{\circ}$ 348/2010. A pesquisa foi realizada no período de outubro a novembro de 2010.

O início da coleta de dados deu-se pelo preenchimento da ficha de identificação do paciente contendo dados sociodemográficos através da escala PDQ-39 e Medida de Independência Funcional (MIF), bem como a partir de entrevistas realizadas, com duração aproximada de 45 minutos. Foram esclarecidas, por parte da pesquisadora, as questões contidas nos instrumentos. Os registros foram expressos em folhas individuais, sendo solicitado aos participantes que respondessem apenas uma das alternativas possíveis.
Instrumentação: O PDQ-39 corresponde a uma escala específica de avaliação da QV na DP que compreende 39 itens distribuídos em oito dimensões: mobilidade (10 itens); atividades de vida diária (AVDs - 6 itens); bem-estar emocional ( 6 itens); estigma (4 itens); suporte social (3 itens); cognição (4 itens); comunicação (3 itens); e, desconforto físico (3 itens). O escore total para cada indivíduo é calculado de acordo com a seguinte fórmula: $100 \mathrm{x}$ (soma dos escores do paciente nas 39 questões / 4 x 39). O escore de cada dimensão é obtido da mesma forma que o escore total. A pontuação total no PDQ-39 varia de 0 (nenhum problema) a 100 (máximo nível do problema), ou seja, uma baixa pontuação indica melhor percepção na QV por parte do indivíduo, sendo que altos escores indicam pobre QV (SOUZA et al., 2007). Essa escala foi validada para o inglês-EUA, inglês-Reino Unido, alemão, espanhol, grego, chinês e francês, sendo traduzida em diversos idiomas, inclusive no português (SANTOS et al., 2010).

A MIF é um instrumento de avaliação que tem como objetivo primordial avaliar, de forma quantitativa, a carga de cuidados demandados por um sujeito para a realização de uma série de tarefas motoras e cognitivas de vida diária, ou seja, as restrições funcionais, de origem variada, observadas em pacientes com doenças neurológicas. Entre as atividades, estão: autocuidados, transferências, locomoção, controle esfincteriano, comunicação e cognição social, que inclui memória, interação social e resolução de problemas. Cada uma das atividades é avaliada e recebe uma pontuação que parte de 1 ponto (dependência total) a 7 pontos (independência completa), as- 
sim, a pontuação total varia de 18 a 126 pontos. Estão descritos dois domínios na MIF, o motor e o cognitivo (RICCI et al., 2006; RICCI; KUBOTA; CORDEIRO, 2005). Esse instrumento de avaliação funcional foi traduzido para a língua portuguesa no Brasil em 2001 e foram realizados testes de reprodutibilidade e confiabilidade, que se mostraram confiáveis para o valor total, bem como nos domínios motor e cognitivo. É importante ressaltar que a MIF não é um instrumento que possa ser autoaplicado (VIANA et al., 2008).

Para análise dos dados, foi utilizado o pacote estatístico SPSS $10.0^{\circledR} \mathrm{e}$ Windows Microsoft Excel ${ }^{\circledR}$, tendo sido analisadas estatísticas descritivas como frequência e média, assim como o Teste de $t$ student. Os dados foram obtidos através da associação entre cada dimensão e o escore total do PDQ-39, além da associação com as seis categorias de atividades propostas pela MIF. Como a maioria das variáveis não apresentou distribuição normal, foi utilizado o teste de Correlação de Spearman a fim de investigar possíveis associações entre estas. Para interpretação dos coeficientes de correlação, o nível de significância considerado foi $\leq 0.05$.

\section{Apresentação e análise dos dados}

Por meio dos dados obtidos na pesquisa, observou-se que dos oito pacientes com doença de Parkinson, cinco eram do sexo masculino e três do sexo feminino, com idades variando entre 59 e 94 anos, com média de 74,87 anos. O'Sullivan e Schmitz (2004) afirmam, com relação ao gênero, que a predominância é no sexo masculino, apontando que os homens apresentam uma incidência ligeiramente maior da doença em relação às mulheres, numa proporção de 3:2. Quanto à comorbidade, seis pacientes apresentaram hipertensão, dois apresentaram diabetes Mellitus e um apresentou cardiopatia. Dados que corroboram com o estudo de Kawasaki (2005) que evidencia que os diagnósticos mais frequentes estão relacionados a doenças do sistema respiratório, cardiovascular, hipertensão arterial sistêmica, seguida de diabetes mellitus. (KAWASAKI; D'ELBOUX, 2005). Com relação ao tabagismo, evidenciou-se que, dos pesquisados, seis sujeitos nunca fumaram, um fumou por 40 anos e outro se autodenominou fumante passivo. No item alcoolismo, seis pacientes responderam que não fazem uso de bebidas alcoólicas. Dos oito sujeitos da amostra, cinco responderam ser sedentários e, na dimensão história familiar, os oito participantes revelaram não ter a doença de Parkinson entre os familiares.

Neste estudo, foram considerados também os estágios da doença, por meio da Escala de Hoehn e Yahr (HY - Degree of Disability Scale). Essa é uma escala de avaliação da incapacidade dos indivíduos com DP, capaz de indicar o estado geral desses sujeitos de forma rápida e prática (LANA et al., 2007). Quanto ao estágio da doença, encontramos os seguintes dados: três pacientes no estágio 1 ; um paciente no estágio 2 ; e, quatro pacientes no estágio 3. As principais características sociodemográficas dos sujeitos da amostra mostraram-se compatíveis com a população que recebeu programas de assistência nas diversas áreas de reabilitação. Dados, estes, semelhantes aos encontrados no estudo de Lana e colaboradores (2007) onde considera os estágios 
da doença do 1 ao 3 como incapacidades leves e moderadas (LANA et al., 2007).

Ao analisar os dados obtidos pelo PDQ-39 observou-se pior percepção da QV nas dimensões "mobilidade", "AVD", "comunicação", "estigma", "bem-estar emocional" e o "apoio social". Percebe-se, no entanto, que os principais sinais e sintomas da DP são motores e que interferem significativamente nas atividades ou tarefas de vida diária, e que os mesmos, somados ao sedentarismo e ao isolamento social, interferem significativamente na $\mathrm{QV}$ dos pacientes (Quadro 1).

Quadro 1. Coeficiente de correlação de Spearman, entre o escore total no PDQ-39 e suas dimensões

\begin{tabular}{|lcc|}
\hline \multicolumn{1}{|c|}{ Dimensão } & $\begin{array}{c}\text { Coeficiente de correlação } \\
\text { de Pearson }\end{array}$ & Classificação \\
\hline Mobilidade & $0,970^{* *}$ & Alta \\
AVD & $0,983^{* *}$ & Alta \\
Bem-estar emocional & $0,897^{* *}$ & Alta \\
Estigma & $0,783^{*}$ & Moderada \\
Apoio social & $0,815^{*}$ & Moderada \\
Cognição & 0,697 & Não significativa \\
Comunicação & $0,870^{* *}$ & Alta \\
Desconforto corporal & 0,576 & Não significativa \\
\hline
\end{tabular}

Nota: ** Correlação significativa ao nível de 0,01. *Correlação significativa ao nível de 0,05.

Em outros estudos, como os demonstrados por Lana et al. (2007), cujo objetivo era avaliar a repercussão dos sinais e sintomas físicos na $\mathrm{QV}$ do indivíduo com $\mathrm{DP}$, os resultados demonstraram que os aspectos físicos podem ser considerados um dos grandes responsáveis pela piora da QV nos indivíduos com DP, pois agem como precursores de limitações em outras dimensões. As limitações físicas certamente afetam emocionalmente o indivíduo, uma vez que esse não consegue realizar suas atividades ocupacionais referentes a mobilidades e AVDs e, consequentemente, perde sua independência funcional (CARNEIRO; FALCONE, 2004).

Em estudos citados por Peto, Jenkinson e Fitzpatrick (1998), que descrevem sobre as áreas afetadas negativamente pela DP, observaram-se alterações significativas correlacionadas a escores específicos de mobilidade e AVD, que corroboram com o presente estudo.

A qualidade de vida relacionada à saúde é vista num contexto abrangente, podendo ser afetada tanto pela saúde física e mental quanto pelo nível de independência funcional, além de trazer consequências para os relacionamentos sociais, crenças pessoais e relações com o meio ambiente (CHRISTOFOLETTI; HYGASHI; GODOY, 2007).

Neste estudo, as dimensões "mobilidade", "AVD", "bem-estar emocional" e "comunicação" apresentaram alta correlação com o escore total do PDQ-39. As dimensões "estigma" e "apoio social" 
também apresentaram moderada correlação com o escore do PDQ-39. Conforme o Quadro 1.

Assim, os aspectos motores e emocionais foram descritos nesta pesquisa como os mais acometidos na DP e, por isso, os domínios ligados a eles podem estar relacionados a uma pior percepção no escore total do PDQ-39. Segundo Neri (2003), a promoção da boa qualidade de vida excede, entretanto, os limites da responsabilidade pessoal e deve ser vista como um empreendimento de caráter sociocultural. Ou seja, resulta das interações que as pessoas buscam, das mudanças e preocupações na sua rotina diária, vislumbrando mais qualidade nas atividades que participam (PASCHOAL, 2006).

Nessa perspectiva, o principal objetivo desse instrumento (MIF), neste estudo, foi avaliar quantitativamente os cuidados que cada paciente requer para a realização de tarefas motoras e cognitivas. Diz respeito, portanto, a uma classificação em termos de dependência ou independência, a qual é baseada na necessidade de ser assistido ou não por outra pessoa e, sendo tal ajuda necessária, em qual proporção. A MIF mede a incapacidade, não a deficiência. Ela tem por objetivo medir o que o indivíduo com incapacidade faz na realidade, não aquilo que ele deveria ou poderia fazer em circunstâncias diferentes (BENVEGNU et al., 2008).

Com relação à MIF, pode-se observar que $49 \%$ dos pacientes analisados apresentou dependência modificada que representa a assistência de até $25 \%$ da tarefa, 38\% apresentou independência completa e $13 \%$ apresentou dependência modificada, ou seja, dependência de até $50 \%$ na realização das tarefas. Como demonstra o Gráfico 1.

Gráfico 1. Medida de Independência Funcional

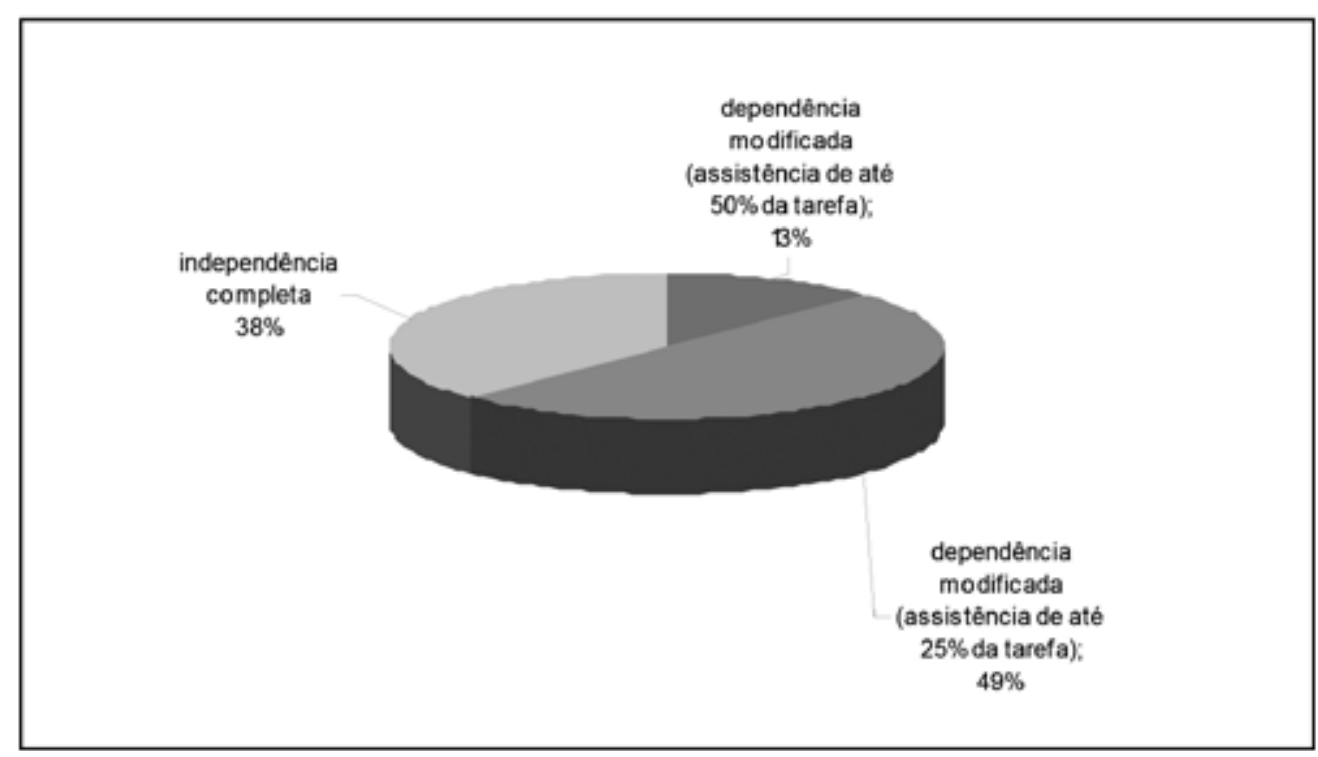


Para esses pacientes, sugere-se atenção dirigida para as áreas de higiene pessoal ou íntima, ao banho, ao vestir-se, à interação social, bem como à resolução de problemas e à memória. Acredita-se que tais áreas devam receber maior ênfase durante os tratamentos com profissionais de equipes multidisciplinares, já que constituem o cotidiano dos pacientes e o intuito desta pesquisa é avaliar qualidade de vida e funcionalidade. Dados semelhantes foram descritos por Ricci (2005), o qual, no item interação social, destaca qie os idosos apresentaram pontuação baixa, necessitando de orientação para lidar com suas próprias necessidades. Kawasaki e D'Elboux (2005) justificam que, no domínio motor, os idosos apresentam um declínio nas tarefas de autocuidado.

Neste estudo, foram encontradas limitações em relação ao tamanho da amostra, dificultando análises mais amplas, e em relação ao estágio da doença, item que não foi levado em consideração para estratificação dos resultados também por estágio.

\section{Considerações finais}

Resultados desta pesquisa indicaram que as limitações motoras relacionadas à mobilidade, AVD, bem-star emocional, comunicação estigma e apoio social possuem associação significativa com a percepção geral da qualidade de vida e funcionalidade dos indivíduos com DP. Esses resultados sugerem que seja realizada uma abordagem por uma equipe multidisciplinar, nos aspectos motores e emocionais, a fim de reestabelecer essas áreas atingidas pela doença de Parkinson, visando a uma melhoria nas atividades diárias, percebida pelo próprio paciente, levando-o a seguir e intensificar as recomendações feitas pelo profissional assistente. $\mathrm{O}$ significado da doença de Parkinson, no que se refere à $\mathrm{QV}$ e à funcionalidade, reveladas neste estudo, fornece dados relevantes no sentido de incentivar programas de reabilitação que sejam apropriados às reais necessidades dos pacientes.

\section{Quality of life and functionin parkinson's disease}

\section{Abstract}

Parkinson's disease (PD) is a neurodegenerative chronic disorder whose diagnosis is established on the basis of clinical symptoms, especially muscle rigidity, resting tremor, bradykinesia and postural abnormalities, which affect aspects of functionality and quality of life of patients this disease. The aim of this work was evaluate the Quality of Life (QOL) and functionality of patients with Parkinson's disease. We studied eight patients with Parkinson's Disease Clinic of Physiotherapy of the University of Passo Fundo. The survey was conducted during October-November 2010. To collect data we used the Questionnaire PDQ 39, FIM (Functional Independence Measure) and an evaluation form neurofuncionnal. The results obtained using the PDQ 39 indicated a poorer perception of QoL in the "mobility" and "Activity of daily living". In MIF, the results indicated that $13 \%$ of the sample requires assistance from $50 \%$ in performing the tasks, 38\% had complete independence and $49 \%$ require assistance from $25 \%$ in performing tasks. After analyzing this study, we can conclude that Parkinson's disease that brings the patient to a functional limitation that interferes with the quality of life.

Keywords: Parkinson's disease. Quality of life. Functionality. Aged. 


\section{Referências}

BEAR, M. F. et al. Neurociências: desvendando o sistema nervoso. 3. ed. Porto Alegre: ARTMED, 2008. 857 p.

BENVEGNU, B. A. et al. Avaliação da medida de independência funcional de indivíduos com seqüelas de acidente vascular encefálico (AVE). Revista Ciência \& Saúde, Porto Alegre, v. 1, n. 2, p. 71-77, jul./dez. 2008.

CARNEIRO, R. S.; FALCONE, E. M. Um estudo das capacidades e deficiências em habilidades sociais na terceira idade. $E s$ tudos em Psicologia, Natal, v. 9, n. 1, p. 119-126, 2004.

CHRISTOFOLETTI, G.; HYGASHI, F.; GODOY, L. R. A. Paralisia cerebral: uma análise do comprometimento motor sobre a qualidade de vida. Fisioterapia em Movimento, Curitiba, v. 20, n. 1, p. 37-44, 2007.

DARABAS, K. C.; COMIM, C. M.; TUON, L. Análise da funcionalidade e qualidade de vida em pacientes portadores de doenças neuromusculares. Fisioterapia Brasil, São Paulo, v. 10, n. 4, p. 241-47, jul./ago. 2009.

FINGER, V. A.; PRADO, C. L. A.; BOFF, M. S. A fisioterapia aquática atuando na melhora das atividades de vida diária em pessoas com doença de Parkinson. Revista da Saúde, Bagé, v. 7, n. 1, p. 107-116, jan./jun. 2003.

FLORIANO, P. J.; DALGALARRONDO, P. Saúde mental, qualidade de vida e religião em idosos de um programa de saúde da família. Journal Brasileiro de Psiquiatria, Rio de Janeiro, v. 53, n. 3, p. 162-170, 2007.

GOULART, F. et al. O impacto de um programa de atividades físicas na QV de DP. Revista Brasileira de Fisioterapia, São Carlos, v. 9, n. 1, p. 49-55, out./dez. 2005.

GUYTON, A. Fisiologia humana e mecanismos das doenças. Rio de Janeiro: Guanabara Koogan, 2008. 639 p.
IKE, D.; CARDOSO, P. N.; BARALDI, I. Análise da incidência de quedas e a influência da fisioterapia no equilíbrio e na instabilidade postural de pacientes com doença de Parkinson. Revista Fisioterapia Brasil, São Paulo, v. 9, n. 1, p. 4-8, jan./fev. 2008.

KAWASAKI, K. M.; D'ELBOUX, J. Impacto da hospitalização na independência funcional do idoso em tratamento clínico. Acta Fisiátrica, São Paulo, v. 12, n. 2, p. 55-60, 2005.

KLINKE, R.; SILBERNAGL, S.; BAUER, C. (Coord.) Tratado de fisiologia. 4. ed. Rio de Janeiro: Guanabara Koogan, 2006. 763 p.

LANA, R. C. et al. Percepção da qualidade de vida de indivíduos com doença de Parkinson através do PDQ-39. Revista Brasileira de Fisioterapia, São Carlos, v. 11, n. 5, p. 397402, set./out. 2007.

LENT, R. 100 bilhões de neurônios: conceitos fundamentais de Neurociência. São Paulo: Atheneu, 2001. p. 454-5.

LUNDY-EKMAN, L. Neurociência - fundamentos para a reabilitação. 3. ed. Rio de Janeiro: Guanabara Koogan, 2000. p. 29.

NERI, A. L. Qualidade de vida e idade madura. 5. ed. Campinas: Papirus, 2003. p. 57-82.

O'SULLIVAN, S. B.; SCHMITZ, T. J. Fisioterapia: avaliação e tratamento. 2. ed. São Paulo: Manole, 2004. 748 p.

PASCHOAL, S. M. P. Qualidade de vida. In: FREITAS, E. V. et al. Tratado de Geriatria e Gerontologia. 2. ed. Rio de Janeiro: Guanabara Koogan, 2006. p. 1040-8.

PETO, V.; JENKINSON, C.; FITZPATRICK, R. PDQ-39: a review of the development, validation and application of a Parkinson's quality of life questionnaire and its associated measures. Journal of Neurology, v. 245, p. 10-14, 1998. Suplemento 1.

PICKLES, B. Fisioterapia na terceira idade. 2. ed. São Paulo: Santos, 2000. p. 81.

POPE, C.; NICHOLAS, M. Pesquisa qualitativa na atenção à saúde. Porto Alegre: Artmed, 2009. p. 11-21. 
RICCI, A. N.; KUBOTA, T. M.; CORDEIRO, C. R. Concordância de observações sobre a capacidade funcional de idosos em assistência domiciliar. Revista de Saúde Pública, São Paulo, v. 39, n. 4, p. 655-662, 2005.

RICCI, N. A. et al. Evolução da independência funcional de idosos atendidos em programa de assistência domiciliária pela óptica do cuidador. Acta Fisiátrica, São Paulo. v. 13, n. 1, p. 26-31, 2006.

SANTOS, V. V. et al. Fisioterapia na doença de Parkinson: uma breve revisão. Revista Brasileira de Neurologia, Rio de Janeiro, v. 46, n. 2, p. 17-25, abr./jun. 2010.

SOUZA, G. R. et al. Quality Of Life Scale In Parkinson's Disease. Arquivos de Neuropsiquiatria, São Paulo, v. 65, n. 3, p. 787-791, 2007.

TEIXEIRA, I. N. D.; NERI, A. L. Envelhecimento bem sucedido: uma meta no curso da vida. Psicologia USP, São Paulo, v. 19, n. 1, p. 81-94, 2008.

VIANA, P. F. et al. Medida de independência funcional nas atividades de vida diária em idosos com sequelas de acidente vascular encefálico no Complexo Gerontológico Sagrada Família de Goiânia. Revista Brasileira de Geriatria e Gerontologia, Rio de Janeiro, v. 11, n. 1, p. 17-28, 2008. 